\title{
Research Article \\ Effect of Polarization Fatigue on Harvesting Energy Using Pyroelectric Materials
}

\author{
Saber Mohammadi \\ Mechanical Department, Razi University, Kermanshah 6714967346, Iran \\ Correspondence should be addressed to Saber Mohammadi; saberm7@yahoo.com
}

Received 13 May 2013; Accepted 11 January 2014; Published 25 February 2014

Academic Editor: Ching-Song Jwo

Copyright (C) 2014 Saber Mohammadi. This is an open access article distributed under the Creative Commons Attribution License, which permits unrestricted use, distribution, and reproduction in any medium, provided the original work is properly cited.

\begin{abstract}
The phenomenon of polarization fatigue in ferroelectric materials is defined and the effect of this phenomenon on harvested energy using these materials has been studied. In order to illustrate this effect, the harvested energy using PZN-4.5PT single crystal was compared in two cases of fatigued and nonfatigued samples. The results have been calculated between two temperatures of 100 and $130^{\circ} \mathrm{C}$ using Ericsson thermodynamic cycle.
\end{abstract}

\section{Introduction}

Ferroelectrics have been the particular popular materials of research for several decades due to their excellent properties which offer potential for development of many modern technological devices, such as microactuators, sensors, and transducers [1-6]. But further studies are necessary to improve the long-time reliability of these devices. A limiting problem to extensive use of ferroelectrics is the suppression of switchable polarization, after certain number of AC drive cycles [5-8]. This is called the polarization fatigue and popularly observed by polarization-electric field (P-E) hysteresis loop measurements. Fatigue in the ferroelectric capacitor corresponds to a decrease of the switchable polarization as a result of bipolar electric field cycling and has been phenomenologically described by many authors $[6,9-12]$. Indeed, polarization in a ferroelectric material is a function of the applied electric field cycle's numbers. Electric field cycling continuously reversing the polarity of the dipoles induces certain changes in a ferroelectric and alters the P-E hysteresis loop. The experimental results on the electrothermal and fatigue characterization of the lead-zirconatetitanate ceramic, PbZr0.53Ti0.47O3 (PZT-5H), for actuator applications have been presented in [13]. Also, fatigue effects due to unipolar cycling of a bulk lead-zirconate-titanate were demonstrated in [14]. Furthermore, polarization and strain hysteresis (P-E and S-E) measurements have been performed simultaneously during fatigue tests under AC fields with a triangular wave form [15].

In the present work, the PZN-4.5PT single crystal that has remarkable pyroelectric properties has been used. Pyroelectric effect converts thermal energy into the electrical current or voltage [16]. This thermal energy can come from many different sources such as ambient temperature, human body temperature, solar energy, and other temperatures of environment. This material possesses several phase changes in the vast range of temperature variations [17]. When the phase change occurred, the sudden increase in current and consequently in polarization is observed. Khodayari et al. [17] show that the phase change increases the harvested energy. Also, it was shown that the results of modeled Ericsson cycle have compatibility with the experimental Ericsson cycle. They measured the value of $216.5 \mathrm{~mJ} / \mathrm{cm}^{3}$ under phase transition using PZN-4.5PT material on the application of Ericsson thermodynamic cycle at 100 and $160^{\circ} \mathrm{C}$. This energy has been calculated on the application of $2 \mathrm{KV} / \mathrm{mm}$ electric field. Guyomar et al. [18] studied the possibilities of harvesting energy from PVDF (polyvinylidene fluoride) film using pyroelectric effect. Materials with high pyroelectric activity or those exhibiting a transition can be used for energy harvesting by converting thermal energy into electrical energy. In this paper, the effect of polarization fatigue on the harvested energy will be studied using modeled thermodynamic Ericsson cycle. 


\section{Pyroelectric Thermodynamic Conversion}

The constitutive equations to describe pyroelectric material are [19]

$$
\begin{gathered}
d D=\varepsilon d E+p d T, \\
d \Gamma=p d E+\frac{\rho c}{T_{0}} d T, \\
p=\left(\frac{d D}{d T}\right), \quad c=\left(\frac{d U}{d T}\right),
\end{gathered}
$$

where $D, E, T, \Gamma, \rho, p, U, \varepsilon$, and $c$ are the electric displacement, electric field, temperature, entropy, mass density, pyroelectric coefficient, internal energy, dielectric coefficient, and thermal capacity, respectively. In order to harvest energy the Ericsson thermodynamic cycle has been used which consists of two constant electric fields and two isothermal processes. Harvested energy is equal to the enclosed area in the polarizationelectric field curve as the following equation [20]:

$$
W_{\text {harvested }}=\oint E d P .
$$

The energy taken from the high temperature reservoir is as the following:

$$
Q_{\text {hot }}=c\left(T_{2}-T_{1}\right)+\int_{0}^{E_{M}} p T_{2} d E,
$$

where $T_{2}$ and $T_{1}$ are the temperatures at high and low reservoir, respectively, and $E_{M}$ is the maximum electric field amplitude. The efficiency is calculated as the following:

$$
\eta=\frac{W_{\text {harvested }}}{Q_{\text {hot }}} .
$$

The electrocaloric effect is very small in the majority of ferroelectric materials, in comparison with thermal capacity multiplied with temperature variations [21], leading to the assumption which is

$$
c\left(T_{2}-T_{1}\right)>>\int_{0}^{E_{M}} p T_{2} d E .
$$

Therefore, the efficiency can be written as the following:

$$
\eta=\frac{W_{\text {harvested }}}{c\left(T_{2}-T_{1}\right)}
$$

\section{Results}

Ozgul and his colleagues [22] present a schematic of the polarization decay in a ferroelectric material as a function of the number of applied electric field cycles. Also, they show that the remnant polarization decreases steadily with increase of the number of cycles. This phenomenon is the polarization fatigue process in the ferroelectric materials. The polarization hysteresis exhibits very slight decreases in the remnant polarization and continuously shifts to lower

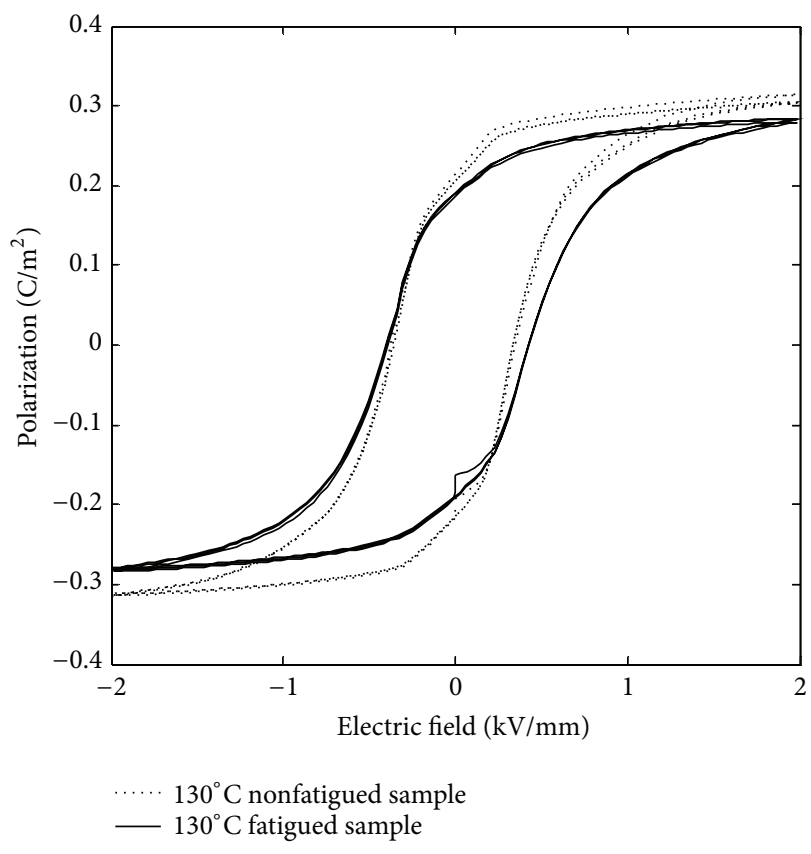

FIGURE 1: Comparison of dynamic hysteresis loops (polarizationelectric field) before (pointed line) and after the fatigue occurrence (continuous line, 600th cycle).

polarization values. These shifts of hysteresis loops display an exponential dependence on the number of measurement cycles. Also, they show that the polarization fatigue is not very perceptible for applied small electric fields, but it began to decrease after about 100 cycles for high electric fields and the fatigue process occurred [22].

In the present work, the bipolar cyclic electric field is applied to the PZN-4.5PT single crystal with dimensions of $8 \times 4 \times 1 \mathrm{~mm}^{3}$ and $8000 \mathrm{~kg} / \mathrm{m}^{3}$ density at $130^{\circ} \mathrm{C}$. Two electrodes of the sample are electrically connected to the power supply and immersed in a bath of silicon oil in order to avoid arcing. This test was performed under application of bipolar triangular voltage waveform switching cycles with amplitude of $2 \mathrm{kV} / \mathrm{mm}$. The cycling field was steadily increased from zero to the maximum field value within $0.25 \mathrm{~s}$; thereafter it was reduced to zero again within $0.25 \mathrm{~s}$. Based on the Ozgul results [22], this field is high and fatigue should occur. Therefore, polarization starts to decrease quickly, approximately after 100 cycles. Figure 1 presents the polarization hysteresis loop versus the electric field for the considered sample, when the sample is not fatigued and then after 600 cycles when the fatigue occurred. The existence of fatigue is confirmed by the shift of polarization hysteresis loop to the lower polarization values as observed. The remnant polarization variations between two cases are about 10 percent.

A pyroelectric converter directly transforms heat energy into electrical energy. Along with the pyroelectric conversion a basic thermodynamic cycle (from the electrical energy producing point of view) will be helpful. The production of the electrical energy may be described in terms of the polarization-electric field behavior of a ferroelectric material. 


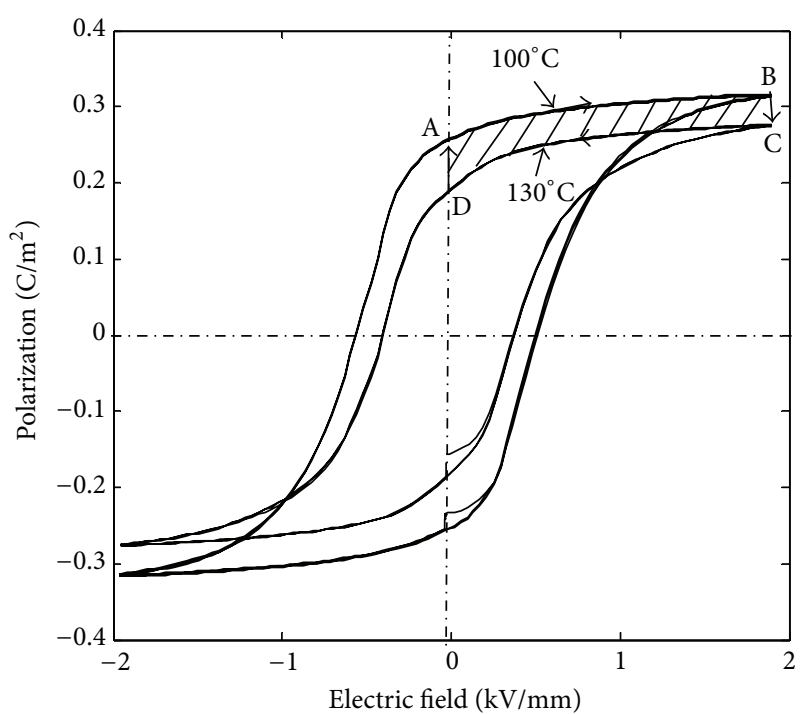

FIGURE 2: Polarization-electric field characteristics of PZT-4.5PT at two different temperatures of 100 and $130^{\circ} \mathrm{C}$.

Figure 2 illustrates P-E (polarization versus applied electric field) behavior of a ferroelectric material which may be utilized for pyroelectric conversion. The area of each cyclic process on P-E diagram represents an electrical work (2). The direction of the path on the cycle (clockwise or counterclockwise) determines whether the electrical energy is produced or dissipated. Electrical energy production cycle may be realized by appropriate phase changes in temperature and applied electric field. Figure 2 is an overlay of the polarization-electric field characteristics of a ferroelectric (PZT-4.5PT) at two different temperatures. It also shows how the clockwise cycling may be achieved when operating is between two temperatures. Starting at point $\mathrm{A}$ at low electric field and low temperature $\left(100^{\circ} \mathrm{C}\right)$ then at this temperature, the ferroelectric (FE) is recharged by increasing the externally applied electric filed until point $B$. Then it is heated at constant electric field until a high temperature $\left(130^{\circ} \mathrm{C}\right)$ (path $\left.\mathrm{BC}\right)$. At this temperature, it is discharged by reducing applied electric field (path CD). Then it is cooled at low electric field (path DA). Therefore, the electrical cycle may be performed in a clockwise manner in which the produced electrical energy per cycle is equal to

$$
W_{\text {harvested }}=\oint E d P=\text { Hatching area }(\mathrm{ABCD}) \text {. }
$$

The cycle is an electrical analogue of the Ericsson thermodynamic cycle.

In this paper, the aim is the effect of polarization fatigue on the harvested energy. Is the fatigue reducing the value of harvested energy? To respond to this question, the Ericsson thermodynamic cycle was modeled with measured polarization at 100 and $130^{\circ} \mathrm{C}$ for the cases in which fatigue occurred and did not occur. The experimental results have been presented in Figure 3. This cycle is an effective thermodynamic cycle and practically feasible [23-26]. It consists of two isothermal and two constant electric field processes. As

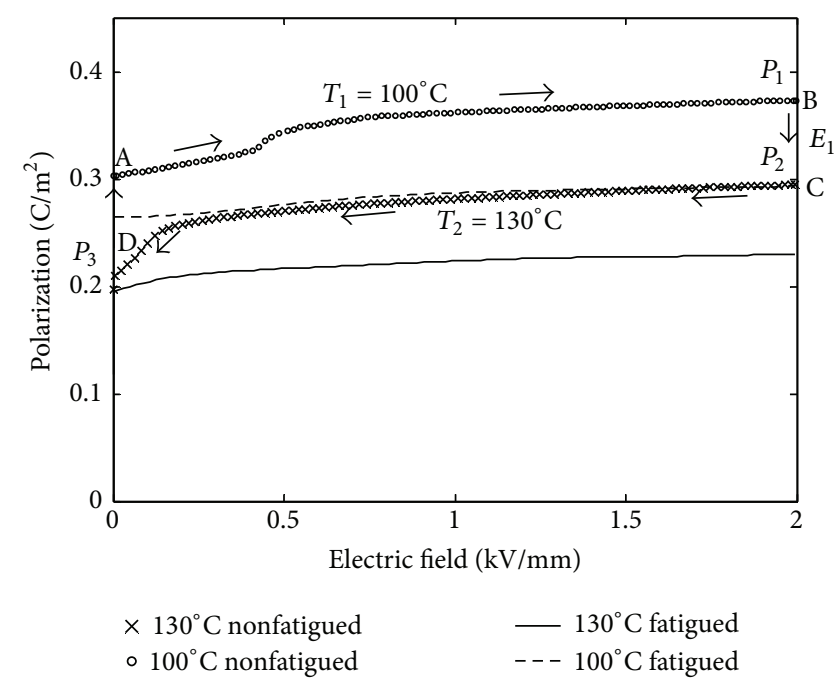

FIgURE 3: Normalized unipolar polarization Ericsson cycle for 2 samples of PZT-4.5PT, fatigued (after 600 cycles) and nonfatigued (after 30 cycles) between 100 and $130^{\circ} \mathrm{C}$.

shown in Figure 3, Ericsson cycle starts at a temperature $T_{1}$, with the application of an increasing electric field until $E_{M}$ $\left(E_{M}=2 \mathrm{kV} / \mathrm{mm}\right)$ which increases the polarization to the value of $P_{1}$ (path $\mathrm{AB}$ ). Temperature is then increased to $T_{2}$ under constant electric field $E_{M}$ (path $\mathrm{BC}$ ) and consequently polarization decreases to $P_{2}$. Then the applied electric field is decreased to 0 which causes a decrease of polarization to $P_{3}$ (path CD). Finally, the temperature is decreased to its initial value at zero electric field (path DA). For more information about modeling of the Ericsson thermodynamic cycle please refer to [17].

The enclosed areas by Ericsson cycles (harvested energy) in two cases of fatigued and nonfatigued are approximately equal and their difference is less than 5\%, although there is a remarkable difference in their curves of polarizationelectric field (Figure 1). The harvested energies in the cases of nonfatigued and fatigued are equal to $20.53 \mathrm{mj} / \mathrm{g}$ and $19.59 \mathrm{mj} / \mathrm{g}$, respectively. Furthermore, the phase change is not observed in the case of fatigued sample. These results indicate that dielectric behavior of ferroelectric materials is undergoing fatigue but their pyroelectric properties is not fatigued.

\section{Conclusions}

The polarization fatigue was observed by polarizationelectric field (P-E) hysteresis loop measurements. Fatigue in the ferroelectric capacitor corresponds to a decrease of the switchable polarization as a result of bipolar electric field cycling. Indeed, polarization in a ferroelectric material is a function of applied electric field cycle's number. Also, the effect of polarization fatigue on the value of harvested energy was studied under applied cyclic switching using modeled thermodynamic Ericsson cycle. According to the results, the polarization fatigue is not very perceptible for applied small electric fields. It is shown that the electrical energy 
production can be described in terms of polarization-electric field behavior of a ferroelectric material. Although there is a remarkable difference between polarization-electric field curves in two cases of fatigued and nonfatigued samples, enclosed areas by Ericsson thermodynamic cycles (the value of harvested energy) in two cases are slightly different. Therefore, the polarization fatigue does not have a serious effect on the value of harvested energy.

\section{Conflict of Interests}

The author declares that there is no conflict of interests regarding the publication of this paper.

\section{References}

[1] K. Uchino, Piezoelectric Actuators and Ultrasonic Motors, Kluwer Academic, Boston, Mass, USA, 1997.

[2] D. L. Polla and L. F. Francis, "Ferroelectric thin films in microelectromechanical systems applications," MRS Bulletin, vol. 21, no. 7, pp. 59-65, 1996.

[3] P. Muralt, A. Kholkin, M. Kohli, and T. Maeder, "Piezoelectric actuation of PZT thin-film diaphragms at static and resonant conditions," Sensors and Actuators A, vol. 53, no. 1-3, pp. 398404, 1996.

[4] Y. Yamashita, "Large electromechanical coupling factors in perovskite binary material system," Japanese Journal of Applied Physics B, vol. 33, no. 9, pp. 5328-5331, 1994.

[5] J. F. Scott, C. A. Araujo, H. B. Meadows, L. D. McMillan, and A. Shawabkeh, "Radiation effects on ferroelectric thin-film memories: retention failure mechanisms," Journal of Applied Physics, vol. 66, no. 3, pp. 1444-1453, 1989.

[6] J. F. Scott and C. A. Paz De Araujo, "Ferroelectric memories," Science, vol. 246, no. 4936, pp. 1400-1405, 1989.

[7] W. L. Warren, B. A. Tuttle, and D. Dimos, "Ferroelectric fatigue in perovskite oxides," Applied Physics Letters, vol. 67, p. 1426, 1995.

[8] A. K. Tagantsev, I. Stolichnov, E. L. Colla, and N. Setter, "Polarization fatigue in ferroelectric films: basic experimental findings, phenomenological scenarios, and microscopic features," Journal of Applied Physics, vol. 90, no. 3, pp. 1387-1402, 2001.

[9] C. A. Paz de Araujo, J. D. Cuchlaro, L. D. McMillan, M. C. Scott, and J. F. Scott, "Fatigue-free ferroelectric capacitors with platinum electrodes," Nature, vol. 374, no. 6523, pp. 627-629, 1995.

[10] W. L. Warren, D. Dimos, B. A. Tuttle, R. D. Nasby, and G. E. Pike, "Electronic domain pinning in $\mathrm{Pb}(\mathrm{Zr}, \mathrm{Ti}) \mathrm{O} 3$ thin films and its role in fatigue," Applied Physics Letters, vol. 65, no. 8, pp. 10181020, 1994.

[11] W. C. Stewart and L. S. Cosentino, "Some optical and electrical switching characteristics of a lead zirconate titanate ferroelectric ceramic," Ferroelectrics, vol. 1, no. 1, pp. 149-167, 1970.

[12] D. B. Fraser and J. R. Maldonado, "Improved aging and switching of lead zirconate-lead titanate ceramics with indium electrodes," Journal of Applied Physics, vol. 41, no. 5, pp. 21722176, 1970.

[13] D. Wang, Y. Fotinich, and G. P. Carman, "Influence of temperature on the electromechanical and fatigue behavior of piezoelectric ceramics," Journal of Applied Physics, vol. 83, no. 10, pp. 5342-5350, 1998.
[14] C. Verdier, D. C. Lupascu, and J. Rödel, "Unipolar fatigue of ferroelectric lead-zirconate-titanate," Journal of the European Ceramic Society, vol. 23, no. 9, pp. 1409-1415, 2003.

[15] M. Ozgul, S. Trolier-Mckinstry, and C. A. Randall, "Fatigue induced effects on bipolar strain loops in PZN-PT piezoelectric single crystals," Journal of Electroceramics, vol. 20, no. 3-4, pp. 133-138, 2008.

[16] W. H. Clingman and R. G. Moore Jr., "Application of ferroelectricity to energy conversion processes," Journal of Applied Physics, vol. 32, no. 4, pp. 675-681, 1961.

[17] A. Khodayari, S. Pruvost, G. Sebald, D. Guyomar, and S. Mohammadi, "Nonlinear pyroelectric energy harvesting from relaxor single crystals," IEEE Transactions on Ultrasonics, Ferroelectrics, and Frequency Control, vol. 56, no. 4, pp. 693-698, 2009.

[18] D. Guyomar, G. Sebald, E. Lefeuvre, and A. Khodayari, “Toward heat energy harvesting using pyroelectric material," Journal of Intelligent Material Systems and Structures, vol. 20, no. 3, pp. 265-271, 2009.

[19] J. Grindlay, An Introduction to the Phenomenological Theory of Ferroelectricity, Pergamon Press, Oxford, UK, 1970.

[20] D. Guyoniar, S. Pruvost, and G. Sebald, "Energy harvesting based on FE-FE transition in ferroelectric single crystals," IEEE Transactions on Ultrasonics, Ferroelectrics, and Frequency Control, vol. 55, no. 2, pp. 279-285, 2008.

[21] G. Sebald, S. Pruvost, and D. Guyomar, "Energy harvesting based on Ericsson pyroelectric cycles in a relaxor ferroelectric ceramic," Smart Materials and Structures, vol. 17, no. 1, Article ID 015012, 2008

[22] M. Ozgul, K. Takemura, S. Trolier-McKinstry, and C. A. Randall, "Polarization fatigue in $\mathrm{Pb}\left(\mathrm{Zn}_{1 / 3} \mathrm{Nb}_{2 / 3}\right) \mathrm{O}_{3}-\mathrm{PbTiO}_{3}$ ferroelectric single crystals," Journal of Applied Physics, vol. 89, no. 9, pp. 5100-5106, 2001.

[23] R. B. Olsen, D. A. Bruno, J. M. Briscoe, and E. W. Jacobs, "Pyroelectric conversion cycle of vinylidene fluoridetrifluoroethylene copolymer," Journal of Applied Physics, vol. 57, no. 11, pp. 5036-5042, 1985.

[24] R. B. Olsen and D. Evans, "Pyroelectric energy conversion: hysteresis loss and temperature sensitivity of a ferroelectric material," Journal of Applied Physics, vol. 54, no. 10, pp. 59415944, 1983.

[25] L. Kouchachvili and M. Ikura, "Pyroelectric conversion-Effects of $\mathrm{P}(\mathrm{VDF}-\mathrm{TrFE})$ preconditioning on power conversion," Journal of Electrostatics, vol. 65, no. 3, pp. 182-188, 2007.

[26] R. B. Olsen and D. D. Brown, "High efficiency direct conversion of heat to electrical energy related pyroelectric measurements," Ferroelectrics, vol. 40, no. 1, pp. 17-27, 1982. 

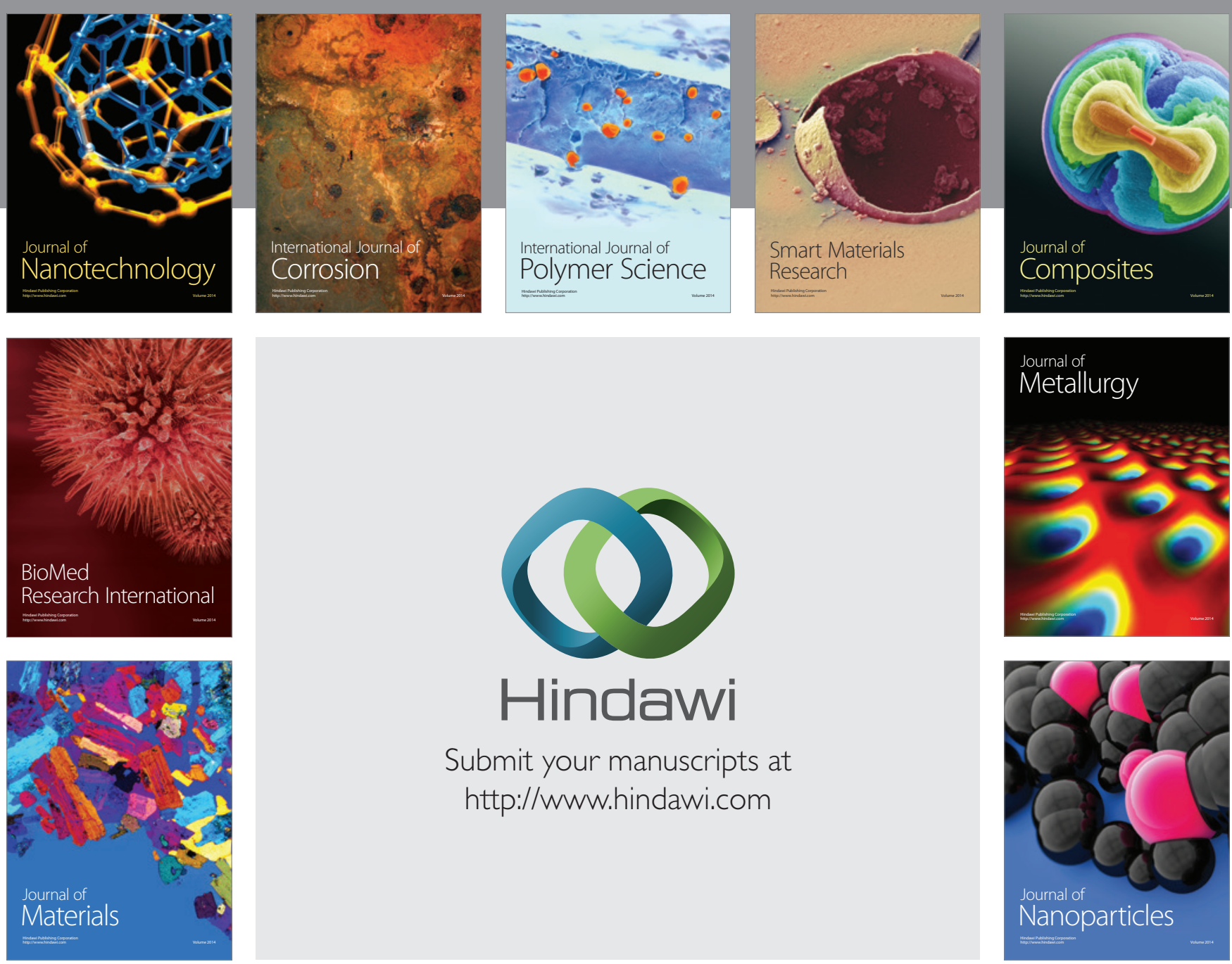

Submit your manuscripts at http://www.hindawi.com
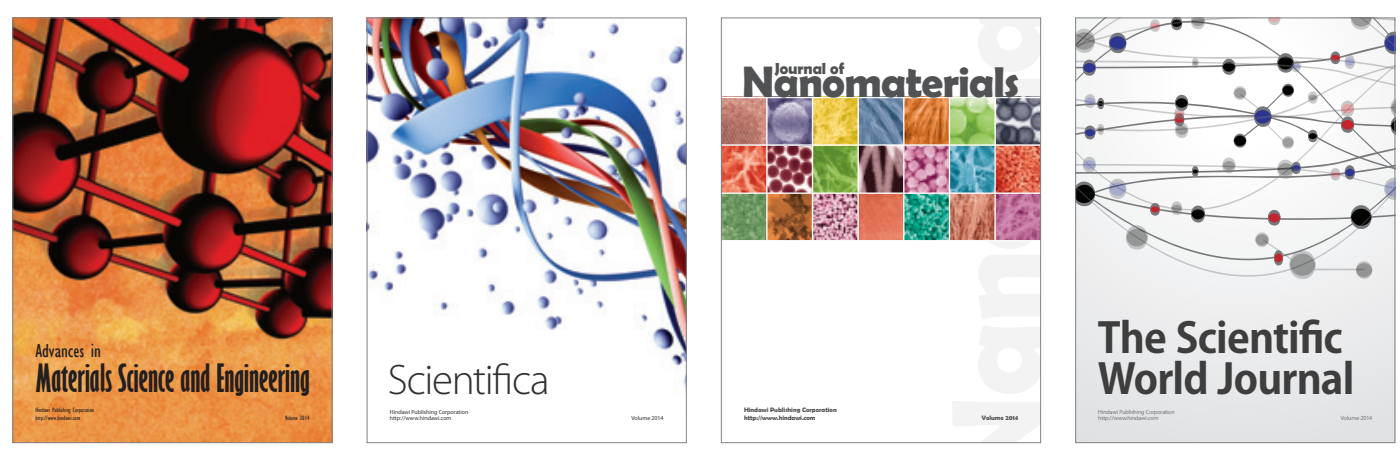

\section{The Scientific World Journal}
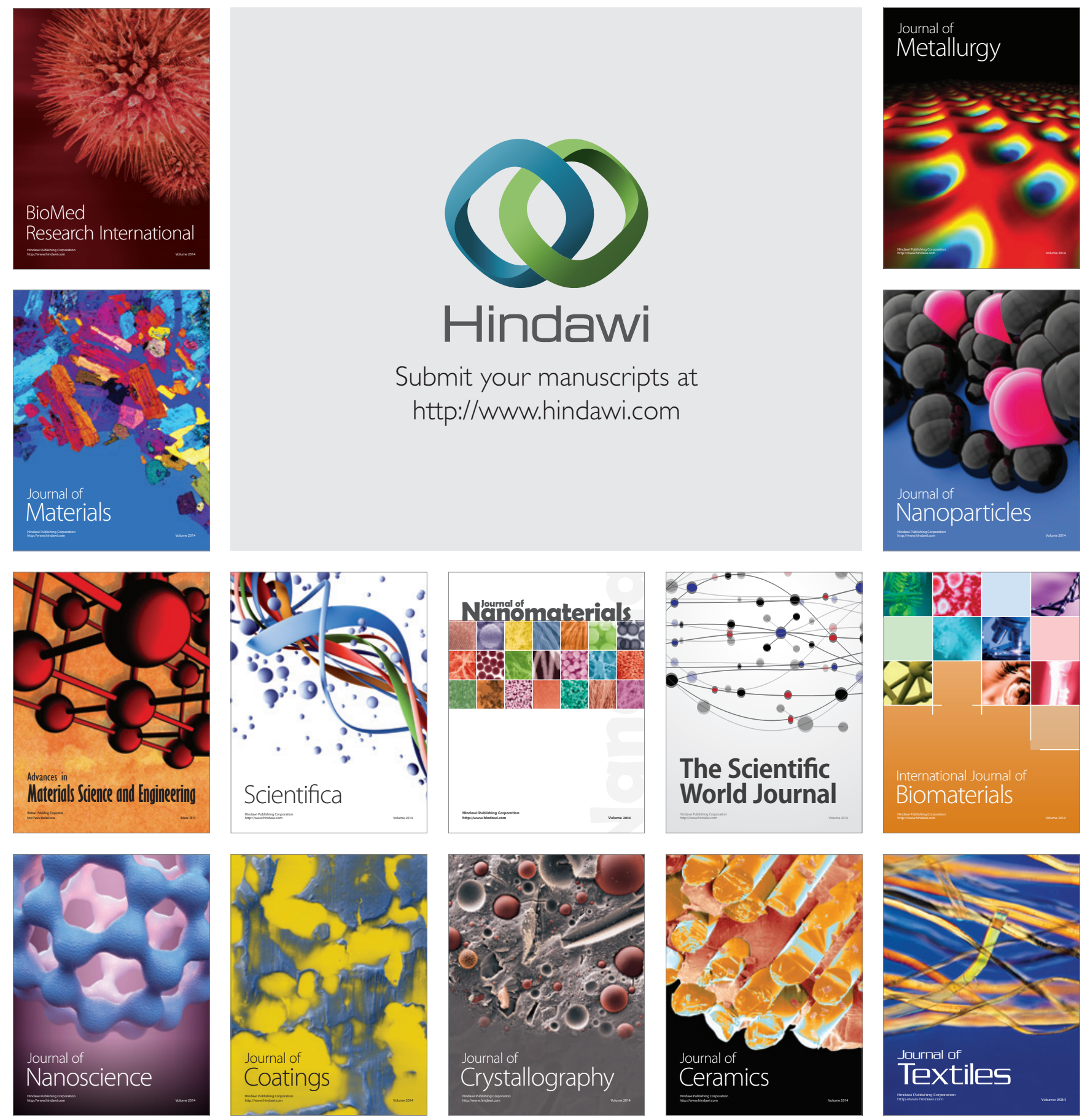